\title{
Contour Extraction Based on Deformable Model and Dynamic Programming
}

\author{
Zhao Cheng' \\ Institute of Manned Space System Engineering, China Academy of Space Technology, Beijing \\ 100094, China;Email:3254059705@qq.com.
}

Keywords: deformable model, region of interest, dynamic programming, contour extraction

\begin{abstract}
In this paper, a new model for extraction of active contours of the quasi-circular targets in a given image which is based on edge detection and dynamic programming(DP) is proposed. In order to guarantee the detected closed contour approximating to the real contour of the target as well as possible, the DP algorithm was launched as the probability distribution of the polar image's edge is obtained by which the computational time was significantly reduced. By the utilization of the image intensity information and the balloon force, the external force in the energy-minimizing function of deformable model is synthetically designed. The theoretical analysis and experiment results demonstrated that the capability of searching a continuous and closed edge contour with the proposed method was remarkably extended compared to the other two methods.
\end{abstract}

\section{Introduction}

The traditional Snake was first introduced by Kass et al. in 1988. Some methods[1] have been proposed to solve this problem, including finite element method, DP algorithm[2-3], greedy algorithm. For example, Amini et al.[4] use the time delayed discrete dynamic programming method to solve the energy minimization problem defined by the ACM. In 2006, H. Zhang et al. [5] propose an automated tongue segmentation method via combining polar edge detector and active contour model. Collewet[6] used a polar description based on orthogonal functions as the parametric curve to describe the contour and introduced an external force derived from an energy term based on the area inside the contour. A detailed investigation on above-mentioned problems is made in this paper, based on which a novel edge extraction method for quasi-circular targets based on deformable model and dynamic programming was designed. The outline of this paper is as follows: The overall contour extraction of targets framework is presented in Section 2, and Section 2 contains edge detection under polar coordinates and building of energy function. In Section 3, we describe the extraction of closed edge contour by using DP. Section 4 shows some experimental results, and the work is summarized and future work is discussed in Section 5.

\section{Deformable Model}

Deformable model mainly consists of two parts: edge information detection and dynamic programming algorithm. Edge information may provide fundamental knowledge for parameterized deformable model, and also prepare for launching DP to obtain optimum, clear, continuous and closed contour. Fig. 1 shows the flow chart of the proposed method.

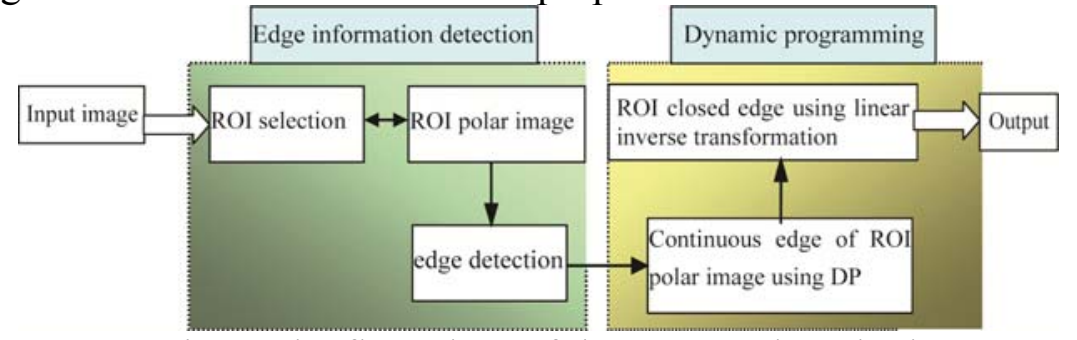

Fig. 1 The flow chart of the proposed method 


\subsection{Edge Detection.}

Fig. 2(a) is a mammogram with benign mass (come from [7]). As showed in Fig. 2, we select a point in mass region and draw the ROI. The blue point is the center point $\left(C_{x}, C_{y}\right)$ and $\mathrm{R}$ is the radius of ROI in Fig. 2(b).
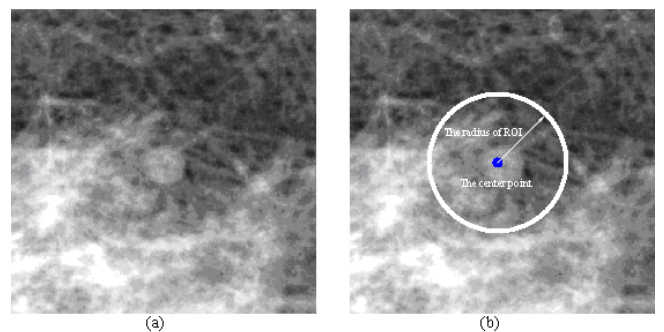

Fig. 2 (a)Mammogram with benign mass (b)Region of interest

We render the center of ROI as the origin of the polar coordinate system $O(r, \theta)$ with the following conditions:

$\left\{\begin{array}{l}x=r \cos \theta \\ x=r \sin \theta\end{array}, \quad 0 \leq r \leq R, 0 \leq \theta \leq 2 \pi\right.$

The ROI $I(x, y)$ shown in Cartesian coordinates was transformed into polar image $I^{\prime}(x, y)$.

\subsection{Engergy Function.}

For a discrete digital image, when it was modeled as a polar image, the radius $r$ and the polar angle $\theta$ can be quantificated with an equidistant interval, and then we can have:

$\begin{cases}r_{i}=i \times(R / M), & i=0,1,2, \ldots M-1 \\ \theta_{j}=j \times(R / N), & j=0,1,2, \ldots N-1\end{cases}$

Thus, a discrete grid or matrix whose sizes are $M \times N$ is obtained. To describe the algorithm in a brief way, $r_{i}\left(\theta_{j}\right)$ is denoted as $r_{i, j}$ for short, and we denote any point $q\left(r_{i}, \theta_{j}\right)$ on the grid as $q_{j}$ similarly as a result of arrangement orderly for candidate points of contour. The optimal path can be expressed with a broken line $B_{N}^{*}=\left\{q_{1}^{*}, q_{2}^{*}, \ldots q_{N}^{*}, q_{1}^{*}\right\}$, which is composed from a candidate point $q_{1}^{*}$ on the first column of matrix to $q_{N}^{*}$ on the last column along the polar angle direction, and come back to $q_{1}^{*}$ at last. The broken line has minimum energy $E\left(B_{N}^{*}\right)$, and it also represents the continuous edge curve of polar image.

In the Snake model, the local energy of any point $q_{j}$ or $q_{j-1} q_{j}$ on the mesh is expressed:

$$
E\left(q_{j}\right)=E_{\text {int }}\left(q_{j}\right)+E_{\text {im }}\left(q_{j}\right)+E_{\text {ext }}\left(q_{j}\right)
$$

where $E_{\text {int }}\left(q_{j}\right)$ is defined as internal energy as follows:

$$
E_{\text {int }}\left(q_{j}\right)=\alpha\left(q_{j}\right)\left|\gamma_{i, j}-\gamma_{k, j-1}\right|+\beta\left(q_{j}\right)\left|\gamma_{i, j}-\gamma_{k, j-1}\right|\left|r_{i, j}+r_{k, j-1}\right| \frac{2 \pi}{N}
$$

where $\alpha\left(q_{j}\right)$ and $\beta\left(q_{j}\right)$ represent parameters of the point $q_{j}$ separately. $E_{i m}$ represents the potential energy result from image force, in which we adopt the normalized gradient magnitude, and it is defined as:

$$
E_{i m}\left(q_{j}\right)=\gamma\left(q_{j}\right) \cdot\left(1-g\left(q_{j}\right)\right) .
$$

$E_{\text {ext }}$ is denoted as external energy which is constituted of the resultant force of the gray external force and the geometric expansive force, which is defined as:

$$
E_{\text {ext }}\left(q_{j}\right)=\mu\left(q_{j}\right) \cdot\left|\operatorname{gray}\left(q_{j}\right)-\operatorname{gray}\left(q_{j-1}\right)\right|+\sigma\left(q_{j}\right) \cdot\left(r_{i, j}+r_{k, j-1}\right)^{2} \cdot \frac{2 \pi}{N}
$$


Where $\mu\left(q_{j}\right)$ and $\sigma\left(q_{j}\right)$ represent the parameters of the point $q_{j}$, and $\operatorname{gray}\left(q_{j}\right)$ represents the gray intensity of the point $q_{j}$. Design of the external energy $E_{\text {ext }}$ is a crucial step in our method for it has a direct impact on the choice of the control points. The details were given as following:

(1) For an image containing quasi-circular targets, it can be divided into three regions: target internal region, background region and target marginal region in which the real contour was involved. When the target ROI was built by $\left(C_{x}, C_{y}\right)$ as the centre of circle and $\mathrm{R}$ as the radius. For example, the points subject to $\left|x-c_{x}\right|^{2}+\left|y-c_{y}\right|^{2} \leq \delta_{1}$ are regarded as target internal region approximately, and the ones restrict to $\delta_{2} \leq\left|x-c_{x}\right|^{2}+\left|y-c_{y}\right|^{2}<R$ belong to background region roughly, where $\delta_{1}, \delta_{2}$ are epsilons. By which the gray mean $m_{\text {int }}$ and the standard deviation $\sigma_{\text {int }}$ of target internal area, the gray mean $m_{\text {back }}$ and the standard deviation $\sigma_{\text {back }}$ of background area can be obtained. What should be stressed is that, prior to the calculation of $m_{\text {int }}$ or $\sigma_{\text {back }}$ etc., only the middle part of the ordered gray value was reserved to eliminate the effect of noise. Then, several rings can be got when the target centroid coordinate $\left(C_{x}, C_{y}\right)$ approximately is regard as center of concentric circles.

(2) According to position of two adjacent control points located in ROI, the dynamic design of the direction of parameters $\mu\left(q_{j}\right), \sigma\left(q_{j}\right)$ in energy term $E_{\text {ext }}$ is shown as follows:

(a)When $q_{j-1}$ locates in background area where it contents with $m_{\text {back }}-3 \sigma_{\text {back }} \leq \operatorname{gray}\left(q_{j-1}\right) \leq m_{\text {back }}+3 \sigma_{\text {back }}$, and $q_{j}$ locates in target internal area depended on $m_{\text {int }}-3 \sigma_{\text {int }} \leq \operatorname{gray}\left(q_{j}\right) \leq m_{\text {int }}+3 \sigma_{\text {int }}$, we set $\mu\left(q_{j}\right):=-\mu\left(q_{j}\right) ; \sigma\left(q_{j}\right):=\sigma\left(q_{j}\right)$. (b)When $q_{j-1}$ locates in target internal area and $q_{j}$ locates in background area, we set $\mu\left(q_{j}\right):=-\mu\left(q_{j}\right) ; \sigma\left(q_{j}\right):=\sigma\left(q_{j}\right)$. (c) When $q_{j-1}$ and $q_{j}$ are both locate in target internal area, we set $\mu\left(q_{j}\right):=\mu\left(q_{j}\right) ; \sigma\left(q_{j}\right):=-\sigma\left(q_{j}\right)$. (d) When $q_{j-1}$ and $q_{j}$ are both locate in background area, we set $\mu\left(q_{j}\right):=\mu\left(q_{j}\right) ; \sigma\left(q_{j}\right):=\sigma\left(q_{j}\right)$, external force plays a positive role while expansibility force turns into shrinkage force. In conclusion, detection of the optimal edge curve $B_{N}^{*}=\left\{q_{1}^{*}, q_{2}^{*}, \ldots q_{N}^{*}, q_{1}^{*}\right\}$ of ROI polar image is equivalent to solve the optimization problem:

$$
\left\{\begin{array}{l}
\min E_{\text {Snake }}=\sum_{j=1}^{N} E\left(q_{j}\right)+E\left(q_{N} q_{1}\right) . \\
\text { s.t. } \quad E\left(q_{j}\right)=0
\end{array}\right.
$$
path.

The solution of problem (7) actually is that we regard target edge detection as searching optimal

\section{Closed Edge Detection by DP}

DP algorithm is an optimization method to deal with multi-step decision process and it is suited to solve the problem (7). For a quasi-circular target trends to be a straight line in polar image, an average filter is cited to detect the potential lines by a convolution operation between the target image and a point spread function (PSF).i.e. $\mathrm{f}^{*}=\mathrm{f} \otimes \mathrm{g}$, where $\mathrm{f}$ represents the target image (shown as Fig. 3(b)), $\mathrm{f}^{*}$ is the filtered image, and $g$ is the PSF.For any point in the filtered image $\mathrm{f}^{*}$, when its gray-scale value is greater than a pre-set value $\delta$, it is chosen as the starting point of DP. Then we can get the starting point sequence $\left\{q_{j}\right\}$, and investigate the distance $d$ between two adjacent starting points $q_{i}$ and $q_{i+1}$. If $\mathrm{d}$ is less than a certain threshold, the point (refers to $q_{i}$ ) was removed otherwise reserved.

\section{Experiment and Analysis}

To illustrate the effectiveness of this algorithm, some images were chosen as the test samples. The parameters were set as following, $\alpha=0.5, \beta=0.05, \gamma=2, \mu=0.001, \sigma=0.01$ in this experiment. The performance of this algorithm was evaluated with overlap percentage calculated 
by Overlap $=$ Area $(A \cap B) / \operatorname{Area}(A \cup B)$, in which A represents the target area and B represents the region after edge extraction, moreover, Area $(X)$ represents the area of region $\mathrm{X}$. When the proposed algorithm was carried out on the images such as bliss image and breast tumor image, the flow chart of detection of edge was shown in Fig. 3 and Fig. 4 from (a) to (f).The results demonstrate that the proposed method achieves satisfactory results.

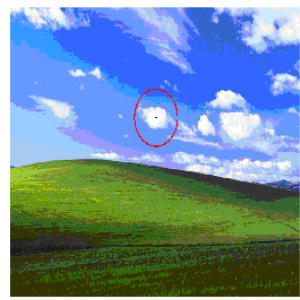

(a) Original Bliss.bmp

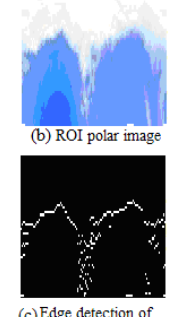

(c) Edge detection of

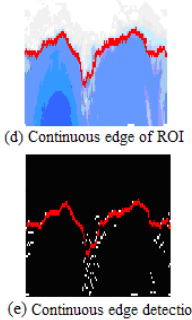

Continuous edge dete
of ROI polar image

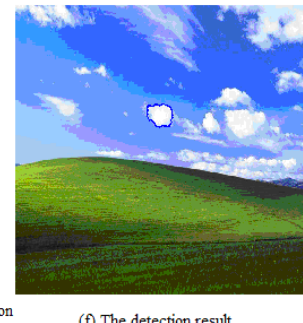

(f) The detection result

Fig. 3 The flow chart of bliss image by the proposed algorithm

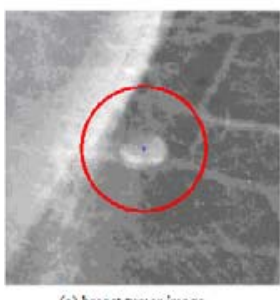

(a) breast tumor image
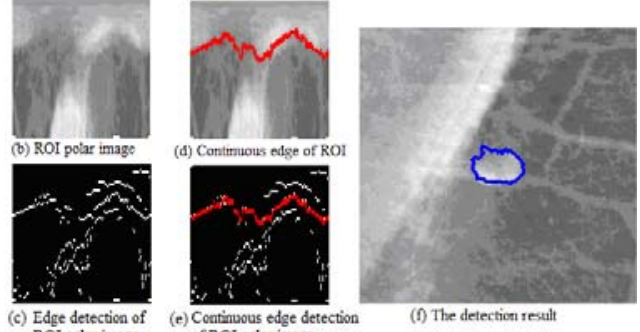

RoI polas imase

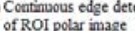

Fig. 4 The flow chart of breast tumor image by the proposed algorithm

Besides, in Fig. 4(a), it can be found that a large area of high-luminance chest rib was involved in ROI which makes the original snake and GVF algorithm trend falling into the local minimum results in a significant deviation between the extracted contour and the real contour.

\section{Conclusion}

In this paper, we have presented a new method for contours extraction of quasi-circular targets which is based on edge information and dynamic programming. The proposed algorithm has strong anti-noise ability and robustness, and the statistics of overlap percentage is significant.

\section{References}

[1] P. Li, T. Zhang. "Review on active contour model (snake model)". J. of softw., vol.11, pp. 751-757, 2000. (in Chinese).

[2] D. Cheng, X. Jiang, "Detections of arterial wall in sonographic artery images using dual dynamic programming,” IEEE Trans. Info. Technol. in Biomed., vol. 12, pp. 792-799, 2008.

[3] C. Sun, P. Vallotton, D. Wang, J. Lopez, Y. Ng, and D. James, "Membrane boundary extraction using circular multiple paths,” Patt. Recogn., vol. 42, pp. 523-30, April 2009.

[4] A.A. Amini, T.E. Weymouth, and R.C. Jain, "Using dynamic programming for solving variational problems in vision,” IEEE Trans. PAMI. vol. 12, pp. 855-67, 1990.

[5] H. Zhang, W. Zuo, K. Wang, and D. Zhang, "A snake-based approach to automated segmentation of tongue image using polar edge detector," Int. J. of Imaging Sys. and Technol., vol. 16, pp. 103-112, 2006.

[6] C. Collewet, "Polar snakes: a fast and robust parametric active contour model," IEEE International Conf. Image Process., pp. 3013-3016, 2009.

[7] S. Timp, and N. Karssemeijer, "A new 2D segmentation method based on dynamic programming applied to computer aided detection in mammography," Med. Phys., vol. 31, pp, 958-71, 2004. 